\title{
Carga de trabalho de enfermagem e sua relação com a gravidade dos pacientes cirúrgicos em UTI*
}

\author{
Relationships among nursing workload, illness severity, and the survival and length of stay of \\ surgical patients in ICUs \\ Carga de trabajo de enfermería y su relación con la gravedad de los pacientes quirúrgicos en uci
}

\section{Alexandre Pazetto Balsanelli ${ }^{1}$, Suely Sueko S. Viski Zanei ${ }^{2}$, Iveth Yamaguchi Whitaker ${ }^{3}$}

\begin{abstract}
RESUMO
Objetivos: Verificar a relação entre a carga de trabalho de enfermagem (TISS-28) e as variáveis: gravidade da doença (APACHE II), sobrevida e, o tempo de permanência na UTI dos pacientes cirúrgicos. Métodos: A coleta de dados realizada de setembro de 2002 a fevereiro de 2003, incluiu 143 pacientes de três UTIs de um Hospital Escola do município de São Paulo. Resultados: os pacientes não sobreviventes obtiveram médias dos escores APACHE II e TISS-28, estatisticamente, mais elevadas quando comparadas às médias dos sobreviventes $(\mathrm{p}=0,001)$. Aqueles que permaneceram por mais tempo nas UTIs obtiveram escores TISS-28 mais elevados, mostrando maior número de horas de cuidados de enfermagem (CE) $(\mathrm{p}=0,005)$. Os pacientes com escores APACHE II mais elevados apresentaram médias de horas de CE estatisticamente superiores $(\mathrm{p}=0,05)$. Conclusões: a carga de trabalho de enfermagem relacionou-se com a gravidade da doença, a sobrevida e o tempo de permanência dos pacientes cirúrgicos na UTI.

Descritores: Carga de trabalho; Tempo de permanência; Cuidados de enfermagem; Cuidados intensivos; APACHE.
\end{abstract}

\begin{abstract}
Objectives: The aim of this study was to analyze the relationships among nursing workload, illness severity, and the survival and the length of stay of surgical patients in ICUs. Methods: Data from 143 inpatients from three ICUs of a teaching hospital in São Paulo city were collected from September 2002 to February 2003. Results: The results showed that patients who did not survive during the length of stay in the ICUs had higher scores on illness severity (APACHE II) and nursing workload (TISS-28) than those individuals who survived ( $\mathrm{p}=$ 0.001). Patients who had longer length of stay in ICUs also had higher scores on nursing workload (TISS-28), which in turn required more hours of nursing care $(\mathrm{p}=0.005)$. And, patients who had a higher score on illness severity (APACHE II) also required more hours of nursing care $(p=0.05)$. Conclusion: nursing workload was related to illness severity and to the survival and length of stay in ICUs.
\end{abstract}

Keywords: Workload; Residence Time; Nursing care; Intensive care; APACHE.

\section{RESUMEN}

Objetivos: verificar la relación entre la carga de trabajo de enfermería (TISS-28) y las variables: gravedad de la enfermedad (APACHE II), supervivencia y, el tiempo de permanencia en la UCI de los pacientes quirúrgicos. Métodos: La recolección de datos realizada de setiembre del 2002 a febrero del 2003, incluyó 143 pacientes de tres UCIs de un Hospital Docente del municipio de Sao Paulo. Resultados: los pacientes no sobrevivientes obtuvieron medias de los escores APACHE II y TISS-28, estadísticamente, más elevadas cuando comparadas a las medias de los sobrevivientes $(\mathrm{p}=0,001)$. Aquellos que permanecieron por más tiempo en las UCIs obtuvieron escores TISS-28 más elevados, mostrando mayor número de horas de cuidados de enfermería (CE) ( $\mathrm{p}=0,005)$. Los pacientes con escores APACHE II más elevados presentaron medias de horas de CE estadísticamente superiores $(\mathrm{p}=0,05)$. Conclusiones: la carga de trabajo de enfermería se relacionó con la gravedad de la enfermedad, la supervivencia y el tiempo de permanencia de los pacientes quirúrgicos en la UCI.

Descriptores: Carga de trabajo; Tiempo de permanencia; Atención de Enfermería; Cuidados Intensivos; APACHE.

*Trabalho de Conclusão do Curso de Especialização em Enfermagem - Modalidade Residência Área de Concentração UTI Adulto do Departamento de Enfermagem da Universidade Federal de São Paulo (SP), Brasil.

${ }^{1}$ Especialista em Enfermagem em UTI pela Universidade Federal de São Paulo (SP), Brasil. Enfermeiro assistencial da Unidade Coronariana do Hospital Israelita Albert Einstein (SP), Brasil.

${ }^{2}$ Enfermeira do Departamento de Enfermagem da Universidade Federal de São Paulo (SP), Brasil. - Área de Enfermagem em Cuidados Intensivos;

${ }^{3}$ Professor Adjunto do Departamento de Enfermagem da Universidade Federal de São Paulo (SP), Brasil. 


\section{INTRODUÇÃO}

As Unidades de Terapia Intensiva (UTIs) constituem setores críticos do hospital destinados aos pacientes graves que necessitam de vigilância contínua e suporte terapêutico especializado. O enfoque interdisciplinar torna-se indispensável para que o paciente tenha suas necessidades de tratamento à saúde atendidas da melhor forma e tão rápido quanto possível.

Dentre as múltiplas admissões existentes nas UTIs encontram-se os pacientes submetidos a cirurgias de grande porte, visto que a recuperação pós-anestésica é realizada nessas unidades, visando não somente sua estabilização, como também o atendimento às possíveis intercorrências decorrentes do estresse cirúrgico que podem ocasionar várias mudanças na homeostase orgânica, tais como: hipotermia, alterações dos níveis pressóricos, arritmias cardíacas, desconforto respiratório, alterações hidroeletrolíticas e ácido-básicas, sangramentos, dentre outras.

Nesse contexto, a equipe de enfermagem dedica elevado número de horas de trabalho na assistência direta ao paciente cirúrgico crítico.

Considerando que o número de procedimentos terapêuticos utilizados para monitorização e tratamento desse tipo de paciente é elevado, os custos hospitalares dessas unidades ganham destaque. Assim, os índices utilizados para estratificar os pacientes de UTI, segundo gravidade e probabilidade de morte, também tornaramse instrumentos de medida que possibilitam, de forma objetiva, avaliar os resultados e investimentos em UTI ${ }^{(1-4)}$.

Um dos instrumentos mais utilizados, para mensurar a gravidade do paciente é o Acute Physiology, Age and Chronic Health Evaluation (APACHE). A versão, denominada APACHE II inclui as seguintes variáveis fisiológicas: temperatura retal, pressão arterial média ou pressão arterial sistólica e diastólica, freqüência cardíaca, freqüência respiratória, oxigenação por meio de valores gasométricos, $\mathrm{pH}$ arterial, sódio, potássio e creatinina séricas, hematócrito e glóbulos brancos, e a escala de coma de Glasgow. Além disso, considera-se a idade cronológica e a presença de doença crônica ${ }^{(1)}$.

Outro índice que foi idealizado para mensurar a gravidade dos pacientes críticos em UTI é o Therapeutic Intervention Scoring System (TISS). Fundamentado na quantidade e tipo de terapêutica implementada para o paciente de UTI, pressupunha-se que quanto mais recursos fossem utilizados, maior seria a gravidade do paciente. No entanto, o desenvolvimento do TISS foi direcionado mais como um instrumento para quantificar a proporção enfermeiro/paciente na UTI do que para mensurar a gravidade do paciente. A versão publicada em 1996 foi denominada de TISS-28 e as intervenções foram agrupadas nas seguintes categorias: atividades básicas, suporte ventilatório, suporte cardiovascular, suporte renal, suporte neurológico, suporte metabólico e intervenções específicas. Assim, considerando que cada ponto do TISS 28 equivale a 10,6 minutos da carga de trabalho de enfermagem por turno nas 24 horas de assistência, tornou-se possível quantificar o número de horas dispensadas à beira do leito para prestação da assistência ao paciente na UTI.

Se, por um lado, a utilização de escalas ou índices são rotineiros na prática médica, em nossa prática diária temos verificado que muitos enfermeiros parecem não valorizar, ou desconhecem a utilização desses instrumentos, bem como há pouca preocupação com os resultados obtidos mediante todos os recursos utilizados na assistência ao paciente grave.

Conhecer os pacientes cirúrgicos admitidos nas UTIs, a gravidade de seu estado - avaliada de forma objetiva, os recursos aplicados, as intercorrências mais comuns,o nível de necessidade de cuidados de enfermagem e o resultado final após a alta, possibilitará descobrir o quanto os tratamentos e cuidados instituídos pela equipe contribuíram para a recuperação dos mesmos.

Considerando-se a complexidade da assistência e tratamentos ministrados ao paciente cirúrgico crítico, questiona-se: qual a carga de trabalho de enfermagem que esse paciente exige na UTI? Existe relação entre a carga de trabalho e a gravidade do paciente, bem como, relação com sua sobrevida e o tempo de permanência na UTI?

Ante o exposto, o objetivo deste estudo consistiu em verificar a relação da carga de trabalho de enfermagem com a gravidade, a sobrevida e o tempo de permanência na UTI dos pacientes cirúrgicos críticos.

\section{MÉTODOS}

Estudo retrospectivo realizado em três UTIs do Hospital São Paulo da Universidade Federal de São Paulo. Trata-se de um hospital escola, de nível terciário, localizado na região sul do município de São Paulo. As UTIs selecionadas, denominadas de Geral, da Pneumologia e Neurocirúrgica atendem pacientes adultos, clínicos e cirúrgicos e totalizaram 27 leitos.

A população foi composta por 143 pacientes cirúrgicos admitidos nessas UTIs que permaneceram internados por, no mínimo, 24 horas, no período de setembro de 2002 a fevereiro de 2003.

Após a aprovação do Comitê de Ética em Pesquisa da Instituição, os dados dos pacientes selecionados foram coletados por meio da consulta aos respectivos prontuários no Serviço de Arquivo Médico.

A coleta de dados foi realizada por meio de instrumento específico para a obtenção de dados fisiológicos das primeiras 24 horas de permanência dos pacientes nas UTIs, para o cálculo do APACHE II, ou 
seja, para mensurar a gravidade do paciente. $\mathrm{O}$ cálculo da carga de trabalho de enfermagem, conforme o TISS 28 , foi realizado com base nos procedimentos realizados, também, nas primeiras $24 \mathrm{~h}$ de internação nas UTIs.

Os demais dados dos pacientes relacionados ao sexo, idade, procedência e procedimento cirúrgico realizado, o tempo de internação nas UTI, o tipo de saída da UTI e após a alta desta unidade também foram coletados.

Para verificar a relação das variáveis estudadas foram utilizados o teste $t$ de Student e a análise de variância pelo método Tukey. Adotou-se um nível de significância de $5 \%$ ou $\mathrm{p}<0,05$.

\section{RESULTADOS}

As características demográficas, procedência, motivo e tempo de internação e condição de saída dos 143 pacientes cirúrgicos estudados estão apresentadas na Tabela 1.

Tabela 1. Pacientes cirúrgicos internados na UTI $(n=143)$ segundo, características demográficas, procedência, motivo e tempo da internação e condição de saída. São Paulo, 2003.

\begin{tabular}{lcc}
\hline \multicolumn{1}{c}{ Variáveis } & \multicolumn{2}{c}{$\begin{array}{c}\text { Pacientes } \\
\text { Cirúrgicos }\end{array}$} \\
& $\mathbf{n}$ & $\mathbf{0}$ \\
& & \\
Sexo: & 62 & 43,4 \\
Feminino & 81 & 56,6 \\
Masculino & & \\
Idade média & 52,7 & $(14 ; 92 ; 18)$ \\
(mínima; máxima; desvio-padrão) & & \\
Procedência: & 127 & 88,8 \\
Centro cirúrgico & 9 & 6,3 \\
Pronto Socorro e Retaguarda & 6 & 4,2 \\
Unidades de Internação & 1 & 0,7 \\
Outra UTI & \multicolumn{2}{c}{} \\
Motivo da internação: & 69 & 48,3 \\
PO neurocirurgia & 23 & 16,1 \\
PO gastrocirurgia & 9 & 6,3 \\
PO transplante Hepático ou renal & 8 & 5,6 \\
PO cirurgia cardiovascular & 8 & 5,6 \\
PO cirurgia torácica & 26 & 18,1 \\
PO de outras cirurgias & 90 & 62,9 \\
Tempo de internação na UTI(até 7 dias) & & \\
Condição de saída & 107 & 74,8 \\
Sobreviventes & 36 & 25,2 \\
Não sobreviventes & & \\
&
\end{tabular}

Conforme os dados apresentados na Tabela 1, a maioria dos pacientes $(56,6 \%)$, era do sexo masculino e a média de idade foi de 52,7 anos.

O Centro Cirúrgico foi a procedência mais freqüente $(88,8 \%)$, sendo que o maior percentual $(48,3 \%)$ relacionou-se ao pós-operatório de cirurgia neurológica, incluindo-se pacientes vítimas de trauma.

A maioria $(62,9 \%)$ dos pacientes permaneceu internada nas UTIs até 7 dias, sendo a média de 10,5 dias (mínimo de um, máximo de 148 e desvio padrão de 18 dias). Do total de pacientes, $36(25,2 \%)$ faleceram nas UTIs.

A média do APACHE II foi de 14,7 pontos e do TISS-28 foi de 27. Destaca-se que 17 pacientes (11,9\%) não tiveram o APACHE II calculado, em decorrência de ausência de registro de dados fisiológicos que compõem este índice, no prontuário do paciente.

Aplicando-se o teste t de Student, observou-se que os pacientes não sobreviventes obtiveram média dos escores APACHE II e TISS-28 estatisticamente $(\mathrm{p}=0,001)$, mais elevados que os sobreviventes; 13,4 e 25,8 pontos do grupo de não sobreviventes e 18,2 e 32,6 pontos dos sobreviventes, respectivamente, indicando gravidade e carga de trabalho de enfermagem mais intensas (Figura 1).

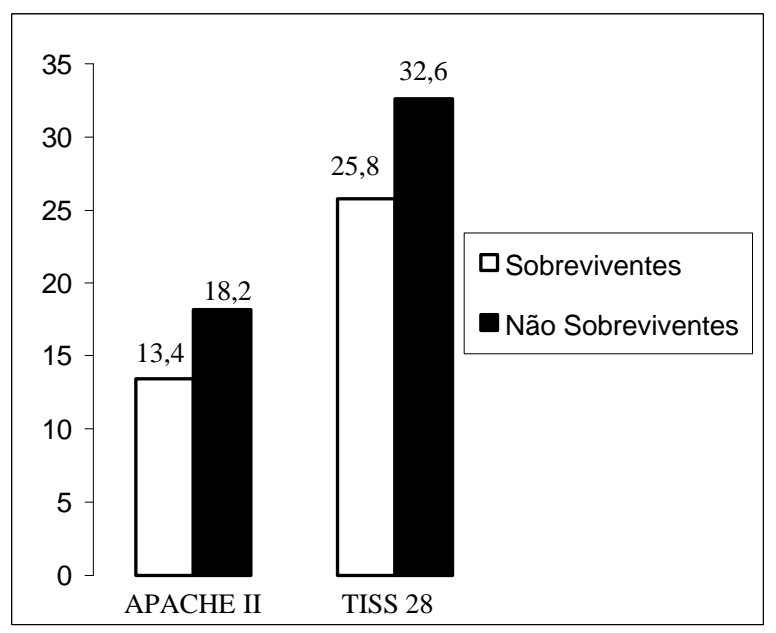

Figura 1- Escores APACHE II e TISS 28 de pacientes cirúricos internados em UTIs segundo condição de saída da UTI. São Paulo, 2003.

$\mathrm{Na}$ tabela a seguir, os dados relativos à média do APACHE II segundo tempo de internação dos pacientes cirúrgicos nas UTIs.

Tabela 2 - Média dos escores APACHE II segundo tempo de internação de pacientes cirúrgicos nas UTIs. São Paulo, 2003.

\begin{tabular}{|c|c|}
\hline $\begin{array}{l}\text { Tempo de internação nas UTIs } \\
\text { (dias) }\end{array}$ & $\begin{array}{c}\text { Média } \\
\text { APACHE II }\end{array}$ \\
\hline Até 7 & 14,2 \\
\hline $8-14$ & 15,1 \\
\hline $15-21$ & 15,8 \\
\hline $22-28$ & 15,7 \\
\hline$>28$ & 11,2 \\
\hline
\end{tabular}

Relacionando-se as variáveis da Tabela 2, não foram encontradas diferenças estatísticas entre elas $(p=0,75)$.

Na Tabela 3, os cálculos das horas de cuidados de enfermagem (H/CE) foram efetuados considerando-se cada ponto do TISS-28 equivalente a 10,6 minutos $^{(5)}$. 
Tabela 3- segundo, Média do TISS-28 e horas de cuidados de enfermagem segundo Tempo de internação de pacientes cirúrgicos nas UTIs. São Paulo, 2003.

\begin{tabular}{|c|c|c|}
\hline $\begin{array}{c}\text { Tempo de internação nas } \\
\text { UTIs } \\
\text { (dias) }\end{array}$ & $\begin{array}{c}\text { Média } \\
\text { TISS 28 } \\
\text { (pontos) }\end{array}$ & $\begin{array}{l}\text { Média } \\
\text { H/CE } \\
\text { (horas) }\end{array}$ \\
\hline Até 7 & 25,9 & 4,6 \\
\hline $8-14$ & 28,7 & 5,1 \\
\hline $15-21$ & 33,6 & 5,9 \\
\hline $22-28$ & 30,6 & 5,4 \\
\hline$>28$ & 30,0 & 5,3 \\
\hline
\end{tabular}

Conforme os dados da Tabela 3, a média do TISS-28 variou de 25,9 a 33,6 pontos e as $\mathrm{H} / \mathrm{CE}$ de 4,6 a 5,9 horas.

Os pacientes que permaneceram mais tempo internados nas UTIs apresentaram o TISS-28 e a média das H/CE estatisticamente, mais elevadas ( $\mathrm{p}=0,002 \mathrm{e} \mathrm{p}=0,005)$.

A classificação da gravidade do paciente, conforme o APACHE II e sua relação com as médias do TISS-28 e horas de cuidados de enfermagem estão apresentadas na Tabela 4.

Tabela 4- Média do TISS-28 e H/CE em pacientes cirúrgicos internados em UTIs segundo o escore APACHE II. São Paulo, 2003.

\begin{tabular}{|c|c|c|c|c|}
\hline APACHE II & $\mathbf{n}$ & $\%$ & $\begin{array}{c}\text { Média } \\
\text { TISS } 28\end{array}$ & $\begin{array}{l}\text { Média } \\
\text { H/CE }\end{array}$ \\
\hline $1-9$ & 21 & 14,7 & 24,9 & 4,5 \\
\hline $10-19$ & 83 & 58,0 & 27,1 & 4,9 \\
\hline $20-29$ & 19 & 13,3 & 28,0 & 5,1 \\
\hline $30 \mathrm{ou}+$ & 3 & 2,1 & 37,0 & 6,5 \\
\hline Dados incompletos & 17 & 11,9 & ----- & --- \\
\hline Total & 143 & 100,0 & & \\
\hline
\end{tabular}

A maioria dos pacientes $(58,0 \%)$ apresentou valores de APACHE II no intervalo de 10 a 19 pontos e, verificase que quanto maior a pontuação deste índice maior é a média do TISS 28 e, conseqüentemente, das horas de cuidados de enfermagem.

\section{DISCUSSÃO}

Quanto aos dados demográficos dos pacientes cirúrgicos internados nas UTIs deste estudo, vale comentar que a idade média da amostra (52,6 anos) é semelhante quando comparada à observada em outros estudos internacionais ${ }^{(6-7)}$ que apresentam média de 59 e 61,5 anos, e nacionais ${ }^{(1,8)}$ que são de 56,0 e 56,9 anos . Além disso, observou-se predomínio do sexo masculino $(56,6 \%)$. A possível justificativa aos resultados obtidos seria que a amostra deste estudo foi constituída exclusivamente de pacientes cirúrgicos, sendo o maior percentual $(48,3 \%)$ relacionado aos pacientes neurocirúrgicos, incluindo-se nesse grupo os pacientes vítimas de trauma, que predominantemente, são jovens do sexo masculino ${ }^{(9)}$.

Em relação ao tempo de permanência nas UTIs, a maioria $(62,9 \%)$ dos pacientes cirúrgicos graves da Instituição permaneceram até sete dias internados nessas unidades. Vale lembrar, que segundo levantamento da Associação de Medicina Intensiva.

Brasileira (AMIB), a média de permanência de 69\% dos pacientes nas UTIs brasileiras é de um a seis dias ${ }^{(10)}$. As UTIs do estudo, portanto, nesse aspecto são semelhantes a realidade nacional. Entretanto, o tempo médio de permanência dos pacientes foi mais elevado em relação a um estudo realizado na Alemanha com 1.986 pacientes admitidos em uma UTI cirúrgica, na qual a média foi de 5,3 dias ${ }^{(4)}$. Todavia, a média do APACHE II desses pacientes foi de 10,3 (DP $=5,2$ ); sendo que no nosso estudo, a maioria $(63,6 \%)$ apresentou um escore médio de 14,2 demonstrando que os pacientes brasileiros, nesse caso, eram mais graves na admissão, o que justificaria um tempo de internação mais prolongado.

Alguns trabalhos ${ }^{(1,11-13)}$ correlacionaram o tempo de permanência e a mortalidade hospitalar, e verificaram que quanto maior o período de internação nas UTIs, maior é a mortalidade dos pacientes. Tal fato explica-se através das complicações que podem advir do tratamento realizado na UTI, destacando-se, principalmente, a exacerbação da doença crônica pré-existente e a infecção hospitalar..

O grupo de pacientes não sobreviventes apresentou média do APACHE II e TISS-28 mais elevada que os sobreviventes $(\mathrm{p}=0,001)$. Isto é corroborado por alguns estudos $^{(2-4,13)}$ que evidenciaram o aumento da pontuação desses escores com a mortalidade. Estudo, realizado no sul do Brasil ${ }^{(1)}$, incluindo 1.411 pacientes cirúrgicos de uma UTI geral apresentou a média do escore APACHE II semelhante ao encontrado no presente estudo, ou seja, 14,1. Ressalta-se que entre os pacientes cirúrgicos a média do APACHE II, bem como a mortalidade, é em geral mais baixa quando comparada aos pacientes clínicos, fato constatado também em outros estudos ${ }^{(1,14)}$.

Por outro lado, o índice APACHE II mais elevado, não foi estatisticamente diferente nos pacientes que permaneceram por mais tempo internados na UTI, $(\mathrm{p}=0,75)$, porém, conforme a Tabela 2 , nota-se que pacientes com longa permanência (entre oito e 28 dias) tendem a apresentar um escore mais elevado. Em estudo cujo foco era analisar a influência do tempo de internação prolongado (maior do que seis dias), o escore APACHE II foi significantemente mais elevado nos pacientes que permaneceram mais tempo na UTI. ${ }^{(7)}$ Essa situação é previsível, considerando-se que sendo mais graves na admissão, a recuperação desses pacientes, conse- 
qüentemente, tende a ser mais demorada, acarretando maior tempo de internação nas UTIs.

Já, quando se relaciona os dias de internação com os valores do TISS $28(p=0,002)$ e as horas de cuidados de enfermagem $(p=0,005)$ nota-se diferença estatisticamente significante, isto é, a necessidade dos cuidados de enfermagem aumenta simultaneamente com o prolongamento dos dias do paciente cirúrgico crítico nas UTIs. Da mesma forma, tal como verificado com o APACHE II, os pacientes que permaneceram nas UTIs por um período entre oito e 28 dias apresentaram pontuação do TISS 28 mais elevada, quando comparados aos pacientes que permaneceram internados até sete dias (Tabela 2 e 3).

Neste estudo, observou-se que o APACHE II teve relação estatisticamente significante $(p=0,05)$ com a pontuação do TISS-28 e as médias das horas de cuidados de enfermagem. Tal fato também foi comprovado em outro estudo $^{(4)}$, que avaliou a aplicação do TISS 28 entre pacientes cirúrgicos críticos: quanto mais altas as pontuações do TISS 28 , maior a gravidade do paciente, segundo o APACHE II.

Nas UTIs, os profissionais de enfermagem constatam, diariamente, que o paciente grave exige maior tempo de assistência, tanto na realização dos procedimentos rotineiros no momento da admissão, como durante sua permanência, em virtude das instabilidades orgânicas que se instalam ao longo de sua permanência nestas unidades.

\section{CONCLUSÃO}

A partir do objetivo proposto, neste estudo,concluiu-se que:

- os pacientes cirúrgicos críticos não sobreviventes das UTIs obtiveram média dos escores APACHE II e TISS28 mais elevada que os sobreviventes $(\mathrm{p}=0,001)$, ou seja, eram mais graves e demandavam maior carga de trabalho de enfermagem;

- os pacientes que permaneceram por mais tempo internados apresentaram o TISS-28 e a média de Horas de Cuidados de Enfermagem (H/CE) significativamente mais elevadas, $\mathrm{p}=0,002 \mathrm{e} \mathrm{p}=0,005$, respectivamente;

- os pacientes com APACHE mais elevado apresentaram médias de $\mathrm{H} / \mathrm{CE}$ estatisticamente superiores $(p=0,05)$.

Assim, considera-se que a aplicação sistemática de índices, tais como os utilizados nesse estudo, pode auxiliar os gerentes de enfermagem das UTIs a dimensionarem o pessoal necessário, visando garantir a melhor assistência possível aos pacientes, com a possibilidade de apresentar os resultados alcançados de forma objetiva.

\section{REFERÊNCIAS}

1. Dias FS, Almeida N, Froemming JB, Guerreiro MO,
Nery PB, Schneider NC.. Avaliação da mortalidade em uma UTI Geral através do escore APACHE II: análise de 1411 casos consecutivos. Rev Med PUCRS. 2001; 11(1):3-8.

2. Cooper GS, Sirio CA, Rotondi AJ, Shepardson LB, Rosenthal GE. Are readmissions to the intensive care unit a useful measure of hospital performance? Med Care. 1999; 37(4):399-408.

3. Wehler M, Martus P, Geise A, Bost A, Mueller A, Hahn EG, Strauss R. Changes in quality of life after medical intensive care. Intensive Care Med. 2001; 27(1):154-9.

4. Chelluri L, Pinsky MR, Donahoe MP, Grenvik A. Longterm outcome of critically ill elderly patients requiring intensive care. JAMA. 1993; 269(24):3119-23.

5. Miranda DR, de Rijk A, Schaufeli W. Simplified Therapeutic Intervention Scoring System: the TISS 28 items - results from a multicenter study. Crit Care Med. 1996; 24(1):64-73.

6. Miranda DR, Ryan DW, Schaufeli WB, Fidler V, editors. Organization and management of intensive care: a prospective study in 12 european countries. Berlin: Springer-Verlag; 1998.

7. Sánchez VL, Reyes SM. Estancia prolongada en terapia intensiva: predicción y consecuencias. Rev Mex Med Crit Ter Int. 2002;16 (2): 41-7.

8. Silva MCM , Souza RMC. Caracterização dos pacientes adultos e adolescentes das unidades de terapia intensiva do município de São Paulo. Rev Paul Enferm. 2002; 21(1): 50-9.

9. Whitaker IY. Gravidade do trauma e probabilidade de sobrevida em pacientes internados [tese]. São Paulo: Escola de Enfermagem da USP; 2000.

10. Associação de Medicina Intensiva Brasileira (AMIB). Anuário Brasileiro de Unidades de Terapia Intensiva. São Paulo; 1999/2000. v.1.

11. Gonçalves WM, Kruel NF, Araujo PA, Teixeira DO. Análise do sistema prognóstico de mortalidade apache II em pacientes cirúrgicos de unidade de terapia intensiva. Rev Col Bras Cir. 1999; 26(2):115-8.

12. Milani Junior R, Rocha AS. Acurácia prognóstica e eficácia de tratamento em UTI avaliadas pelo sistema APACHE II. Rev Hosp Clin Fac Med Univ São Paulo. 1989; 44(4):149-52.

13. Rodrigues AT, Pitol R, Camillo CJ, Cerutti LA, Silva S. Aplicação do índice prognóstico Apache II em Unidade de Terapia Intensiva Geral. J Bras Med. 2001; 80(4):36-8.

14. Bersi F, Zanei SSSV, Whitaker IY. Pacientes clínicos internados em unidades de Terapia Intensiva de um hospital escola: seguimento da admissão a alta hospitalar [resumo]. In: $11^{\circ}$ Congresso Brasileiro de Medicina Intensiva, 2004 Out 23. Curitiba; Anais. Curitiba; 2004. p.174. 